\title{
Gram per Milliliter per Kilogram per Meter Squared
}

National Cancer Institute

\section{Source}

National Cancer Institute. Gram per Milliliter per Kilogram per Meter Squared. NCI

Thesaurus. Code C119352.

A unit of concentration equal to grams per milliliter, divided by kilograms per meter squared. 